TITLE:

\title{
Electrochemical stabilization of porous silicon multilayers for sensing various chemical compounds
}

\section{AUTHOR(S):}

Salem, MS; Sailor, MJ; Harraz, FA; Sakka, T; Ogata, $\mathrm{YH}$

\section{CITATION:}

Salem, MS ...[et al]. Electrochemical stabilization of porous silicon multilayers for sensing various chemical compounds. JOURNAL OF APPLIED PHYSICS 2006, 100 (8): 083520.

\section{ISSUE DATE:}

2006-10-15

URL:

http://hdl.handle.net/2433/50430

\section{RIGHT:}

Copyright 2006 American Institute of Physics. This article may be downloaded for personal use only. Any other use requires prior permission of the author and the American Institute of Physics. 


\title{
Electrochemical stabilization of porous silicon multilayers for sensing various chemical compounds
}

\author{
M. S. Salem \\ Institute of Advanced Energy, Kyoto University, Uji, Kyoto 611-0011, Japan \\ M. J. Sailor \\ Department of Chemistry and Biochemistry, University of California, San Diego, California 92039-0358 \\ F. A. Harraz, ${ }^{\text {a) }}$ T. Sakka, and Y. H. Ogata ${ }^{\text {b) }}$ \\ Institute of Advanced Energy, Kyoto University, Uji, Kyoto 611-0011, Japan
}

(Received 28 March 2006; accepted 31 July 2006; published online 30 October 2006)

\begin{abstract}
Porous silicon rugate filters are fabricated and investigated for their ability to sense chemical species. The durability of the filter is tested by allowing the structure to undergo many cycles of adsorption and desorption of vapor-phase ethanol molecules. The characteristic reflectivity peak of the structure exhibits a relative blueshift of $2.7 \%$ after 86 adsorption/desorption cycles. The observed shift is ascribed to the formation of silicon dioxide, which has a lower refractive index than that of silicon. In order to stabilize the structure against oxidation expected from cycling and environmental exposure, the filter is subjected to electrochemical oxidation in an aqueous sulfuric acid electrolyte. The treatment dramatically improves stability of the sensor; a relative blueshift of $<0.4 \%$ is observed after 100 adsorption/desorption cycles for this sensor. The sensitivity of the sensor is also affected by electrochemical oxidation: the response to saturated ethanol in air changes from $\Delta \lambda=100 \mathrm{~nm}$ to $\Delta \lambda=70 \mathrm{~nm}$, respectively. Theoretical calculations using the Bruggeman effective medium approximation and the characteristic matrix method indicate that up to $15 \%$ (by volume) of silicon is transformed to silicon dioxide by the electrochemical oxidation procedure. This volume ratio is close to that estimated from Auger electron spectroscopy measurements. (C) 2006 American Institute of Physics. [DOI: 10.1063/1.2360389]
\end{abstract}

\section{INTRODUCTION}

The optical or electrical properties of porous silicon ${ }^{1,2}$ (PS) single layers have been used in many chemical and biological sensing applications due to the large specific surface area of the material $\left(200-800 \mathrm{~m}^{2} / \mathrm{cm}^{3}\right)$. The adsorption of chemical or biological substances into the pores modifies the electrical ${ }^{3,4}$ and optical ${ }^{5,6}$ properties of porous silicon, allowing convenient and sensitive measurement of bonding events. The high surface area of porous silicon also provides a convenient means of immobilizing a large amount of various biomolecules including enzymes, ${ }^{7}$ DNA fragments, ${ }^{8}$ and antibodies ${ }^{9}$ in a relatively small area.

Porous silicon is typically prepared by electrochemical anodization of crystalline silicon in hydrofluoric (HF) acid solution. The anodization current density determines the porosity, which is the volumetric fraction of the pores inside the structure. The higher the porosity, the lower is the refractive index. It is generally accepted that the electrochemical etching process in the silicon/fluoride system is self-limiting and occurs only at the interface between porous $\mathrm{Si}$ and crystalline $\mathrm{Si}$ substrate, that is, the already etched porous layer is unaffected by further electrochemical etching. ${ }^{10,11}$ Thus the porosity can be modulated in depth. ${ }^{12,13}$ This allows the fabri-

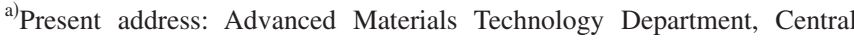
Metallurgical Research and Development Institute, Helwan, Cairo 11421, Egypt.

b) Author to whom correspondence should be addressed; electronic mail: y-ogata@iae.kyoto-u.ac.jp
}

cation of PS multilayers with a variety of refractive index profiles. This is a rapid and inexpensive means to fabricate one-dimensional photonic structures. The spectral peak position of the multilayer is a strong function of the average refractive indices of the layers, and a shift in the photonic feature has been shown to provide a very sensitive method for detection of condensable vapors ${ }^{14,15}$ and other molecules that can enter the pores. This method can also be applied for the detection of explosives ${ }^{16}$ and chemical weapons. ${ }^{17}$

A rugate filter ${ }^{18}$ prepared by sinusoidally modulating current density during anodization of silicon exhibits a characteristic spectral peak in the reflectivity spectrum. The porous silicon rugate filter (PSRF) has similar filter characteristics compared to conventional porous silicon multilayers based on a Bragg stack optical structure. ${ }^{19}$ Rugate filters possess a gradual variation in porosity, while the porosity in porous silicon Bragg reflectors varies abruptly layer by layer. In a Bragg stack, the individual layers must be designed such that they are phase matched, while rugate filters do not have this requirement and so they are simpler to fabricate. ${ }^{20}$

The mechanical and chemical stability of PSRFs is an important issue, especially for applications in which the material is thermally cycled or repeatedly flushed with liquid. Oxidation is one of the most common stabilization methods. It imparts a hydrophilic character to the surface which allows the structure to sense hydrophilic molecules that are miscible with water. In addition, the temperature dependence of silicon refractive index in the visible and near infrared regions causes the resonant wavelength of silicon-based photonic 
structures to redshift upon heating. ${ }^{21}$ The surface stress induced by oxide coverage on a silicon matrix has been shown to counterbalance this effect and reduce the temperature dependence of the photonic crystal resonant. ${ }^{22}$ The method employed here involves electrochemical oxidation, which has the advantage that it is relatively easy to incorporate into the porous silicon manufacturing process. Thermal oxidation of porous silicon multilayers ${ }^{23}$ is widely used to produce stable interference filters, but this method has been shown to collapse the pore structure in some cases. ${ }^{24}$ Various oxidative methods to improve the mechanical weakness have been investigated. ${ }^{25,26}$ Electrochemical oxidation, on the other hand, can be easily controlled by the amount of charge passed, which is proportional to the amount of oxide formed.

In this study we discuss some parameters that produce degradation in the spectral line shape of PSRFs employed for sensing various chemical species. We focus on the electrochemical oxidation of PSRFs as an efficient and easy technique to stabilize the filters. Another objective of this study is to try to clarify the mechanism of electrochemical oxide growth in PSRFs.

\section{EXPERIMENT}

\section{A. Sample preparation}

A PSRF was prepared by electrochemical anodization of a (100)-oriented boron-doped $p$-type silicon wafer (0.01-0.02 $\Omega \mathrm{cm}$ in resistivity) in $22 \mathrm{wt} \%$ HF ethanolic solution. Prior to anodization, the silicon wafer was dipped in 5 wt \% HF solution to remove the native oxide. The electrochemical anodization cell made of trifluoroethylene resin was employed with an $\mathrm{O}$ ring at the bottom that allowed an effective electrode area of $0.79 \mathrm{~cm}^{2}$. Current density, supplied by a programmable current source (Keithly 6221), was sinusoidally oscillated between 68 and $94 \mathrm{~mA} \mathrm{~cm}^{-2}$. After performing anodization, the sample was dried under a stream of dry argon gas.

Two porous silicon single layers were prepared by applying current densities at the highest and lowest values of the current signal applied for the etching process. The porosity, $P_{\text {in }}$, of each layer was gravimetrically estimated. ${ }^{27}$ Porosity values of $53 \%$ and $42 \%$ were obtained corresponding to the higher and lower current densities, respectively. Since the average pore size is much smaller than the incident visible wavelength used in this study, the refractive index of the prepared single layers can be estimated using Bruggeman's effective-medium-approximation relationship ${ }^{14}$ given by

$$
P_{\text {in }} \frac{n_{\text {air }}^{2}-n_{\mathrm{eff}}^{2}}{n_{\text {air }}^{2}+2 n_{\mathrm{eff}}^{2}}+\left(1-P_{\mathrm{in}}\right) \frac{n_{\mathrm{Si}}^{2}-n_{\mathrm{eff}}^{2}}{n_{\mathrm{Si}}^{2}+2 n_{\mathrm{eff}}^{2}}=0,
$$

where $n_{\text {air }}, n_{\mathrm{Si}}$, and $n_{\text {eff }}$ are the refractive indices of air, silicon, and the effective porous medium, respectively. Refractive indices of 2.15 and 2.44, corresponding to the lower $\left(n_{L}\right)$ and higher $\left(n_{H}\right)$ limiting refractive indices, were obtained using Eq. (1). It should be pointed out that the Bruggeman model used in this work is a simple effective medium approximation. However, this simple model is applicable to porous materials with moderate porosity such as porous sili- con prepared from heavily doped silicon, as described by Theiss. ${ }^{28}$

\section{B. Experimental setup for durability investigation}

An experimental setup was employed to check the durability of the fabricated PSRFs. The sample was placed in a cell into which air saturated with ethanol vapor was introduced. The cell had an optical window to measure reflectance spectra in situ. An electric current was allowed to pass through the silicon substrate to heat the sample. The reflectance spectra were in situ collected during repeated cycling of the electric current between on and off states under continuous exposure to the saturated vapor.

\section{Electrochemical oxidation}

The electrochemical oxidation of the PSRF was carried out in a three-electrode cell connected to an automatic polarization system (HZ-3000, Hokuto Denko). The filter acted as the working electrode. The counterelectrode was a platinum rod, whereas $\mathrm{Ag} / \mathrm{AgCl}$ was used as the reference electrode. Anodic oxidation was performed in $1 \mathrm{M} \mathrm{H}_{2} \mathrm{SO}_{4}$ under galvanostatic conditions of $2.5 \mathrm{~mA} \mathrm{~cm}{ }^{-2}$ and different oxidation times.

\section{Characterization}

A tungsten-halogen lamp was used as a source of illumination and the reflectance spectra were measured using a spectrograph equipped with a charge-coupled device (CCD) detector. Reflection-mode fourier transform infrared (FTIR) spectra were recorded in dried air environment by means of a spectrometer (Jasco FT-IR-460). A silicon wafer was used as reference. The data were averaged over 64 scans with a resolution of $4 \mathrm{~cm}^{-1}$.

Scanning electron microscopy (SEM) (JSM-5600 JEOL) was used to observe the porosity variation in the direction of pore propagation and to determine the thickness of the porous film. Auger electron spectroscopy (AES) (BJAMP-7810 JEOL) provided an elemental analysis over the whole cross section of the filter.

\section{RESULTS AND DISCUSSION}

\section{A. Fabrication and characterization of PSRF}

A sinusoidal current oscillating between 68 and $94 \mathrm{~mA} \mathrm{~cm}^{-2}$ was applied for $5 \mathrm{~min}$ to prepare a PSRF. This condition produced a multilayer of $11.5 \mu \mathrm{m}$ in thickness with an average apparent pore size of $25 \mathrm{~nm}$. PSRFs having different periodicities were prepared by changing the periodic time of the current signal used in the anodization process. Figure 1 shows the dependence of the resonant wavelength on the period of the electric current wave form. Resonant wavelengths of 453, 545, and $673 \mathrm{~nm}$ were obtained corresponding to periodicities of $0.09,0.11$, and $0.15 \mu \mathrm{m}$, respectively. The resonant feature is determined by the periodicity and the refractive index gradient of the structure. ${ }^{29}$ The cross-sectional SEM image of PSRF whose resonant wavelength is located at $545 \mathrm{~nm}$ is depicted in Fig. 2 . This image shows the porosity variation in the direction of 


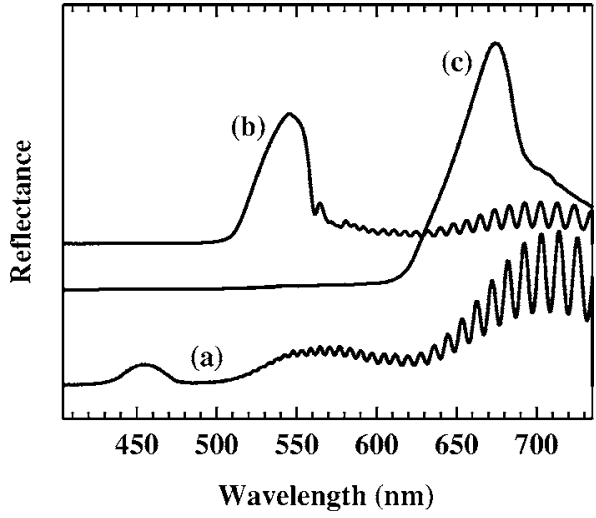

FIG. 1. Reflectance spectra of PSRFs prepared by electrochemical anodization of highly doped silicon using a sinusoidal current oscillating between 68 and $94 \mathrm{~mA} \mathrm{~cm}^{-2}$ for $5 \mathrm{~min}$ at different current signal periodic times: (a) 2.3 , (b) 3.0 , and (c) $4.0 \mathrm{~s}$.

pore propagation. When this structure is exposed to condensable vapor such as ethanol or methanol, a capillary condensation of vapor to liquid displaces the air in the nanometersized pores. ${ }^{30}$ The condensation causes an increase in the average refractive index of the matrix, which results in a significant redshift in the reflectivity spectra, as displayed in Fig. 3. This behavior provides a general, nonspecific means to sense various volatile chemical species. The obtained redshift correlates with the refractive index and the vapor pressure of the analyte.

\section{B. Durability for repeated use}

The stability of PSRF is an essential issue for repeated use in environmental sensing applications. The stability of an as-prepared sample was investigated using the setup described in the Experiment section. An as-prepared PSRF was maintained under continuous exposure to ethanol-saturated air. Electric current was applied through the silicon substrate and varied between on and off states. The resulting resistive heating allows the structure to undergo many adsorptiondesorption cycles of ethanol molecules. When electric cur-

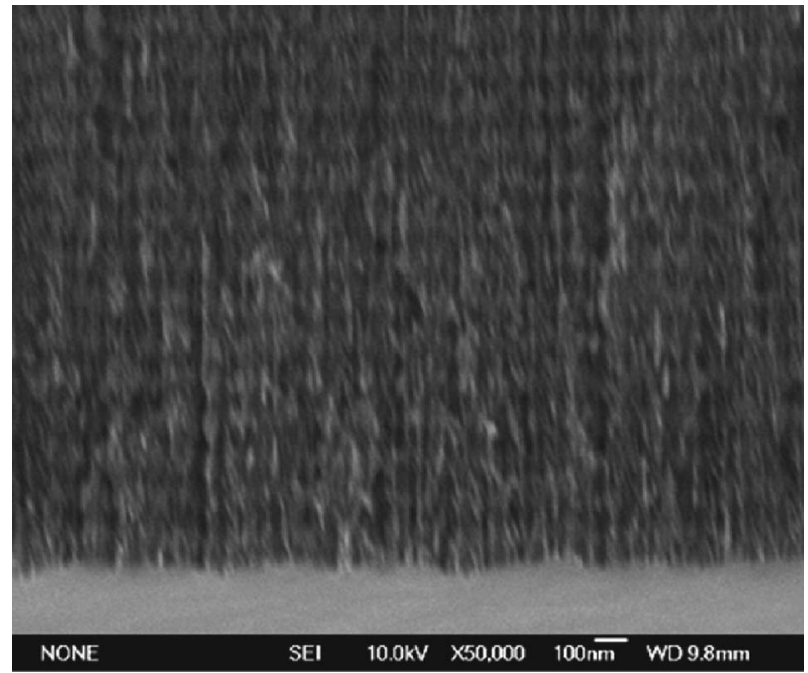

FIG. 2. Cross-sectional view of PSRF whose resonant wavelength is located at $545 \mathrm{~nm}$.

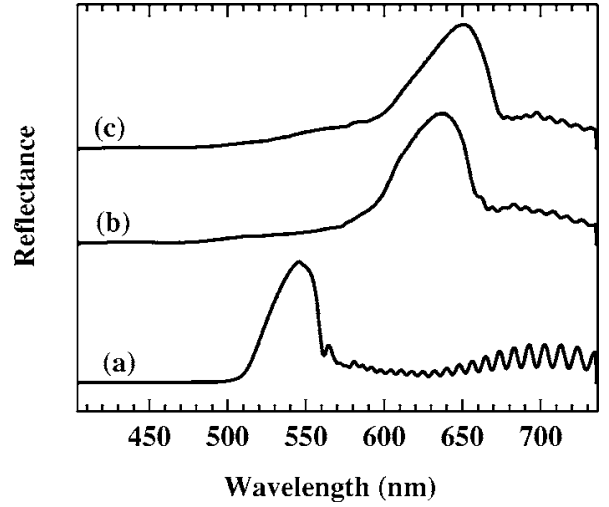

FIG. 3. Reflectance spectra of PSRFs before (a) and after exposure to saturated vapors of methanol (b) and ethanol (c) for $15 \mathrm{~min}$.

rent was turned off, an observable redshift was detected, as shown in Fig. 4, due to the capillary condensation of ethanol vapor into the pores and the subsequent increase in the average refractive index of the filter as it cools down. By switching on the current, resistive heating causes a complete drying of the pores due to the thermal evaporation of ethanol molecules and shifts the resonant wavelength back to its original (in air) position. It is found that a relative blueshift of $2.7 \%$ from the original peak wavelength is observed after 86 cycles of the durability test. This blueshift is attributed to the oxidation of the multilayer.

FTIR spectra (data are not shown here) were recorded before and after 86 cycles of the durability test. FTIR of a freshly prepared filter shows the $\mathrm{Si}-\mathrm{H}$ stretching modes and other features characteristic of a typical as-prepared porous silicon single layer. ${ }^{31}$ After 86 cycles of the durability test, a loss in the intensity of the $\mathrm{Si}-\mathrm{H}_{x}$ stretching modes and complete disappearance of $\mathrm{Si}-\mathrm{H}_{2}$ scissors mode were observed. In addition, three further absorption bands are observed at 850,1100 , and $2927 \mathrm{~cm}^{-1}$. The former two bands are assigned to stretching vibrations of silicon oxide, and the peak at $2927 \mathrm{~cm}^{-1}$ is attributed to the $\mathrm{C}-\mathrm{H}$ stretching modes of ethanol. ${ }^{32}$ These results indicate that an oxidation of the PSRF occurs after repeated cycling in an oxygen-containing

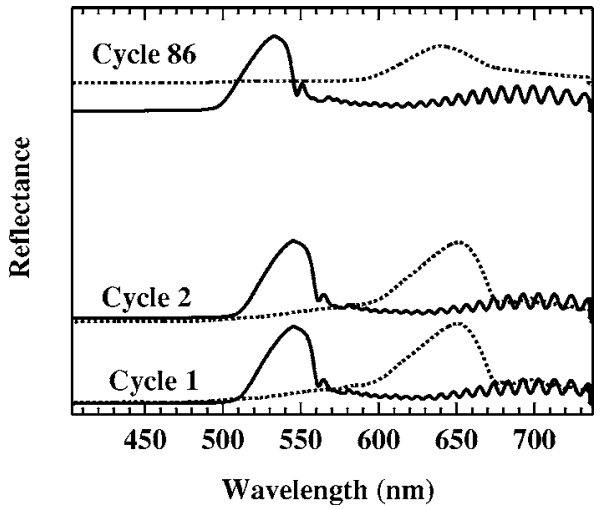

FIG. 4. Stability acceleration test of as-prepared PSRF. The characteristic peak of the structure (solid curves) exhibits a blueshift after 86 cycles of ethanol vapor adsorption-desorption. The dashed curves obtained by switching off the electric current show a redshift after adsorption of ethanol molecules into the pores. 


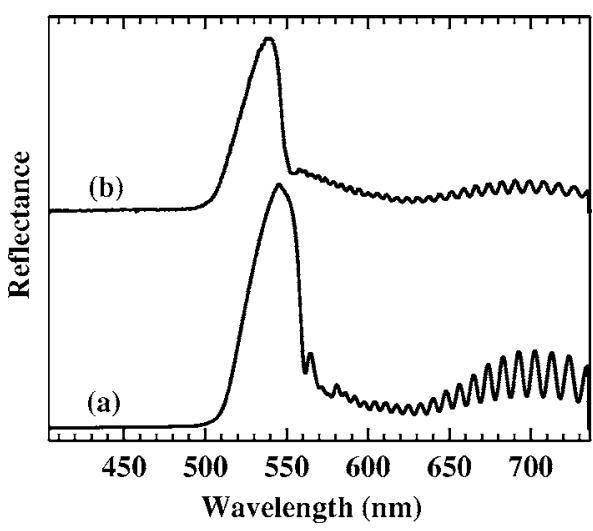

FIG. 5. Reflectance spectrum of PSRF (a) and that recorded after storing the filter in ambient air for 4 months (b).

environment and that ethanol molecules become either chemisorbed or strongly physisorbed to the silicon oxide surface.

\section{Aging of PSRF}

The effects of environmental aging are of importance in the practical applications of porous silicon multilayers. The growth of native oxide in an uncontrolled manner leads to zero-point drift in the reflectivity spectrum that are not acceptable for most sensor applications. A relative blueshift of $1.4 \%$ in the resonant wavelength of the PSRF was observed due to storage in ambient conditions for four months, as shown Fig. 5. This can be interpreted as the transformation of part of silicon to silicon oxides which have a refractive index lower than that of silicon. In a related work, ${ }^{33,34}$ the resonant wavelength of a porous silicon multilayer prepared from lightly doped silicon exhibited a blueshift greater than 4\% in 100 days of the storage in ambient atmosphere. The difference between our results and the previously reported results is most likely related to the different doping level of the silicon substrates, different levels of ambient oxidants such as nitrogen oxides and ozone, and uncontrolled changes in the relative humidity. The highly doped silicon used in the present study produces a mesoporous structure which has a slower oxidation rate than that of the microporous structure obtained from lightly doped silicon due to its lower specific surface area. ${ }^{35,36}$ Aging builds oxides from upper to lower layers with an oxidation rate proportional to the specific surface area and the thickness of the multilayer. This effect makes the freshly prepared porous silicon multilayers unsuitable for most environmental sensing applications.

\section{Electrochemical oxidation}

A PSRF was electrochemically oxidized to avoid uncontrolled oxidation caused by natural aging and to enable the repeated use in oxygen-containing samples. The electrochemical oxidation of a PSRF was conducted in $1 \mathrm{M} \mathrm{H}_{2} \mathrm{SO}_{4}$ at current density of $2 \mathrm{~mA} \mathrm{~cm}^{-2}$. The potential transient during oxidation exhibited the same behavior as in the case of the single layer. ${ }^{37}$ An almost constant potential was observed initially, followed by a slight increase in potential with increasing oxidation time. The oxidation reaction is generally

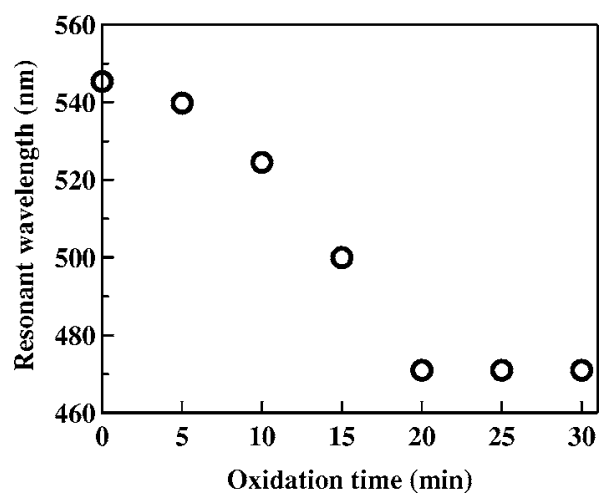

FIG. 6. Variation of resonant wavelength of as-prepared PSRF with the electrochemical oxidation time.

limited by hole concentration at the silicon surface. Holes are available preferentially at the pore bottom, and oxidation initiates in this region. Once the pore bottom becomes covered by an oxide layer, feedback control in the galvanostat increases the applied potential to maintain constant current density. Oxidation then proceeds through the pore walls toward the top of the porous film. The oxidation process stops before complete oxidation of the multilayer because the electrical contact between the bulk silicon and the pore tip of the lowest porous layer is broken by the oxide. This situation corresponds to a sudden rise of the electrode potential observed at long oxidation time. Thus the anodization process becomes self-limiting.

The FTIR spectra of PSRF were observed at different stages of oxidation time. These results indicate that oxidation generates mainly $\mathrm{SiO}-\mathrm{H}, \mathrm{O}_{y}=\mathrm{Si}-\mathrm{H}_{x}$, and $\mathrm{Si}-\mathrm{O}-\mathrm{Si}$ absorption bands ${ }^{31,38}$ and leaves behind some $\mathrm{Si}-\mathrm{H}_{x}$ surface species. The remaining $\mathrm{Si}-\mathrm{H}_{x}$ species observed provides evidence that some fraction of the silicon nanocrystallites are unaffected by the oxidation process.

The evolution of the resonant wavelength of the PSRF with oxidation time is depicted in Fig. 6. As the oxidation time increases, the degree of oxidation increases and the resonant wavelength shifts toward the blue end of the spectrum. Oxidation replaces silicon nanocrystallites with silicon oxide, which has a refractive index lower than that of silicon. The resonant wavelength displays no further blueshift at oxidation time longer than $\sim 20 \mathrm{~min}$. As mentioned above, the self-limiting nature of the electrochemical oxidation process results in only a fraction of silicon being transformed to silicon dioxide. The fraction of the oxidized silicon was investigated by AES. Figure 7 shows the AES spectrum averaged over the whole thickness of a PSRF anodically oxidized at $2.5 \mathrm{~mA} \mathrm{~cm}^{-2}$ for $20 \mathrm{~min}$. The volumetric fraction of silicon which has been converted to silicon dioxide at an oxidation time of $20 \mathrm{~min}$ is estimated as $14.1 \%$ using the spectrum shown in Fig. 7.

The durability of the oxidized structure for sensing ethanol vapor-saturated air was investigated. It was found that the oxidized structure endured more than 100 adsorptiondesorption cycles in ethanol vapor without any detectable change in the resonant wavelength $(<0.4 \mathrm{~nm})$ and characteristic peak shape. The reflectance spectrum of the oxidized filter was obtained again after maintaining the filter in an 


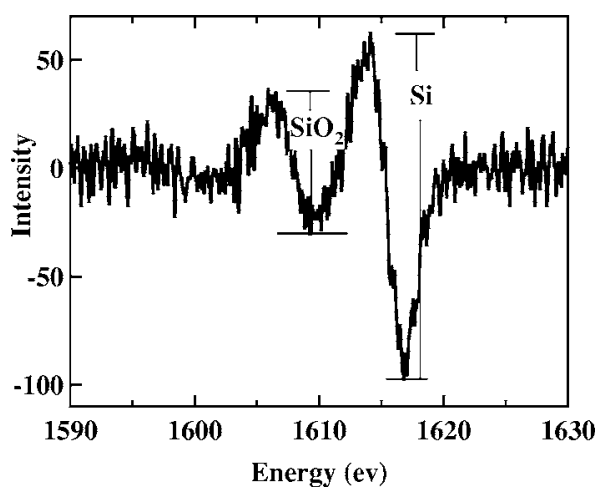

FIG. 7. Differential mode AES spectrum of PSRF anodically oxidized in $1 \mathrm{M} \mathrm{H}_{2} \mathrm{SO}_{4}$ under galvanostatic conditions of $2.5 \mathrm{~mA} \mathrm{~cm}^{-2}$ for $20 \mathrm{~min}$. The spectrum is averaged over the cross section of the filter. The peak intensities of Auger $K L L$ electrons of silicon and silicon dioxide are indicated.

ambient air environment for 6 months. No degradation of the characteristic resonant peak was observed during and after this time period. It seems that electrochemical oxidation renders the filter stable enough to be employed in environmental sensing applications. It is worth mentioning that chemical functionalization by grafting of organic groups to porous silicon via $\mathrm{Si}-\mathrm{C}$ bonds has also been implemented to protect the material from natural oxidation. ${ }^{39,40}$

The relative sensitivity of the filter decreases somewhat on oxidation. The freshly prepared ( $\mathrm{Si}-\mathrm{H}$ terminated) PSRF exhibits a redshift in the resonant of $\sim 100 \mathrm{~nm}$ when exposed to ethanol-saturated air, while the electrochemically oxidized PSRF exhibits a shift of only $\sim 70 \mathrm{~nm}$.

Table I compares the change in the resonant wavelength of the oxidized PSRF after infiltrating various liquids into the pores and exposing the structure to saturated vapor of each liquid. The steady state was reached after $15 \mathrm{~min}$ of exposure to saturated vapor for all the analytes studied. Liquids used in this study are methanol, acetonitrile, ethanol, 2-propanol, $n$-heptane, and ethylene dichloride whose refractive indices are $1.32,1.34,1.36,1.37,1.39$, and 1.42 , respectively. The redshift produced after exposure to saturated vapors of ethanol and methanol is smaller than that obtained in Fig. 3 using as-prepared PSRF. This is attributed to the lower average refractive index of oxidized PSRF, which provides a smaller change in average refractive index of the matrix upon liquid

TABLE I. Resonant peak position of oxidized PSRF centered at $470 \mathrm{~nm}$ after exposure to saturated vapor of some liquid and filling the pore with liquid.

\begin{tabular}{cccc}
\hline \hline & $\begin{array}{c}\text { Saturated vapor } \\
\text { pressure (torr) } \\
\text { at 20 C }\end{array}$ & $\begin{array}{c}\text { Resonant } \\
\text { wavelength } \\
\text { (nm) after } \\
\text { exposure to } \\
\text { saturated vapor }\end{array}$ & $\begin{array}{c}\text { Resonant } \\
\text { wavelength } \\
\text { (nm) after } \\
\text { completely filling } \\
\text { the pore } \\
\text { with liquid }\end{array}$ \\
\hline Methanol & 96 & 524 & 544.2 \\
Acetonitrile & 73 & 533 & 546.5 \\
Ethanol & 43 & 538 & 549.5 \\
2-propanol & 33 & 549 & 557.2 \\
$n$-heptane & 40 & 556 & 560.3 \\
Ethylene dichloride & 61 & 562 & 569 \\
\hline \hline
\end{tabular}

infiltration into the pores. The peak positions after the pores are completely filled with liquid are also given in the table.

Table I implies that a partial substitution of air with liquid occurs when exposing the filter to saturated vapors and completely liquid-filled pores cannot be obtained via capillary condensation. The result is in agreement with Snow et al. ${ }^{14}$ and contradicts the study by Mulloni and Pavesi ${ }^{41}$ which reported $100 \%$ filled pores due to capillary condensation of liquids into the pores. This contradiction can be explained by the use of different types of silicon substrates. Mulloni and Pavesi fabricated their multilayer structure (a Bragg stack), from lightly doped silicon. This low doping level produces a microporous material (pores $<2 \mathrm{~nm}$ in diameter) which allows microcapillary condensation to occur in a much larger fraction of the porous film. The oxidized structure was also exposed to saturated vapor of dichloromethane which has almost the same refractive index as ethanol. The redshift produced due to the condensation of dichloromethane molecules was smaller than that of ethanol. The former liquid has higher vapor pressure (381 torr at $20^{\circ} \mathrm{C}$ ) which results in difficult condensation into the pores. ${ }^{30}$

\section{E. Simulation for PSRF design and oxidation}

The refractive index profile of a rugate filter sinosoidally oscillating between $n_{L}$ and $n_{H}$ can be represented as ${ }^{42}$

$$
n(X)=N_{\mathrm{av}}+\frac{1}{2} N_{\text {peak }} \sin \left(\frac{4 \pi N_{\mathrm{av}} X}{\lambda_{0}}\right),
$$

where $N_{\text {peak }}$ is the peak-to-peak index variation, $n_{H}-n_{L}, N_{\text {av }}$ is the average index of the filter, $\frac{1}{2}\left(n_{L}+n_{H}\right), X$ is the film thickness, and $\lambda_{0}$ is the central wavelength of the filter. The theoretical reflectivity spectrum was calculated based on the transfer matrix method. ${ }^{43}$ The sinusoidal variation of refractive index was treated as discrete layers. We divided each cycle of the refractive index profile into different numbers of layers and calculated the reflectivity spectrum in each case until the result became independent of the number of layers. We found that dividing each cycle of the index profile into more than 23 layers has no significant effect on the calculated spectrum. Wavelength dependence of silicon refractive index was taken into consideration during calculations. Figure 8 compares the theoretical calculations to the experimentally obtained results. The experimental result shows a slight redshift in the peak position compared to the theoretically predicted position. This can be attributed to a deviation from the ideal sinusoidal variation in porosity assumed in our model. This depth inhomogeneity is more important for porous silicon multilayers with larger numbers of repeating layers. ${ }^{44}$ The theoretical calculations display large sidelobes around the stop band. This can be suppressed during preparation of the films by including some matching layers and an apodization function to the etching wave form. ${ }^{45}$ In addition, the sidelobes can be suppressed if the porous layer has significant absorption of light in the region of the spectrum of interest.

After anodic oxidation, the structure of porous silicon is no longer a mixture of silicon and air. A third component in 


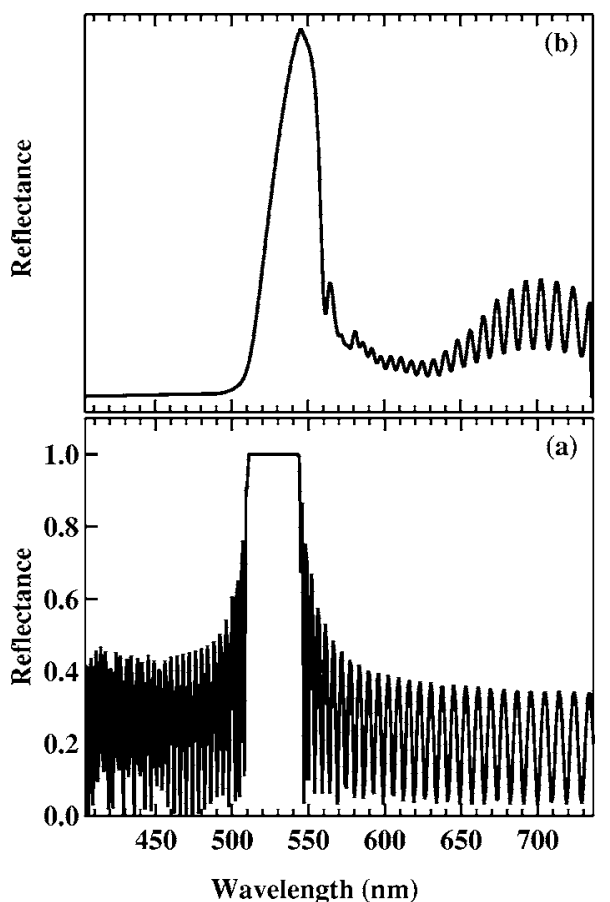

FIG. 8. Simulated reflectivity spectrum of PSRF (a) and the experimentally recorded one (b). The filter was prepared by electrochemical anodization of highly doped silicon using a sinusoidal current wave form oscillating between 68 and $94 \mathrm{~mA} \mathrm{~cm}^{-2}$ for $5 \mathrm{~min}$ with a periodic time of $3 \mathrm{~s}$.

the form of silicon dioxide whose refractive index, $n_{\mathrm{SiO}_{2}}$ is ca. 1.46 should be involved. Three component Bruggeman formula, Eq. (3), has to replace Eq. (1).

$F_{\mathrm{Si}} \frac{n_{\mathrm{Si}}^{2}-n_{\mathrm{eff}}^{2}}{n_{\mathrm{Si}}^{2}+2 n_{\mathrm{eff}}^{2}}+F_{f} \frac{n_{\mathrm{air}}^{2}-n_{\mathrm{eff}}^{2}}{n_{\mathrm{air}}^{2}+2 n_{\mathrm{eff}}^{2}}+F_{\mathrm{SiO}_{2}} \frac{n_{\mathrm{SiO}_{2}}{ }^{2}-n_{\mathrm{eff}}^{2}}{n_{\mathrm{SiO}_{2}}^{2}+2 n_{\mathrm{eff}}^{2}}=0$

where $F_{\mathrm{SiO}_{2}}$ is the volume fraction of silicon dioxide, whereas $F_{f}$ and $F_{\mathrm{Si}}$ are the fractions of air and silicon remaining after oxidation. Assuming that some portion of silicon, $x$, has been transformed to silicon dioxide, the remaining volume fraction of silicon after oxidation, $F_{\mathrm{Si}}$, is $1-P_{\text {in }}$ $-x$, where $P_{\text {in }}$ is the initial porosity prior to oxidation. Since the density of silicon and silicon dioxide has nearly the same value $\left(2.3\right.$ and $\left.2.2 \mathrm{~g} / \mathrm{cm}^{3}\right)$, addition of $2 \mathrm{~mol}$ of oxygen for every mole of silicon results in a 2.27 -fold increase in volume of the solid portion of the matrix. This volume increase produces a silicon dioxide volume fraction of $2.27 x$. The remaining volume fraction in the oxidized structure accounts for the change in porosity upon oxidation. At a very high degree of oxidation, the volume increase of the solid matrix becomes higher and leads to the collapse of the porous structure. Based on these assumptions, the Bruggeman equation for the oxidized porous silicon layer can be written as:

$$
\begin{aligned}
& \left(1-P_{\mathrm{in}}-x\right) \frac{n_{\mathrm{Si}}^{2}-n_{\mathrm{eff}}^{2}}{n_{\mathrm{Si}^{2}}{ }^{2}+2 n_{\mathrm{eff}}^{2}}+\left(P_{\mathrm{in}}-1.27 x\right) \frac{n_{\mathrm{air}}{ }^{2}-n_{\mathrm{eff}}^{2}}{n_{\mathrm{air}}{ }^{2}+2 n_{\mathrm{eff}}^{2}} \\
& +2.27 x \frac{n_{\mathrm{SiO}_{2}}{ }^{2}-n_{\mathrm{eff}}^{2}}{n_{\mathrm{SiO}_{2}}{ }^{2}+2 n_{\mathrm{eff}}^{2}}=0 .
\end{aligned}
$$

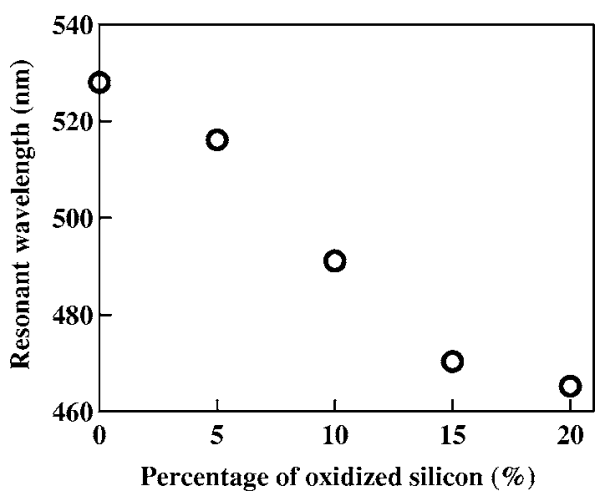

FIG. 9. Simulated resonant wavelength of oxidized PSRF at different volume fractions of oxidized silicon.

The change in the lower and upper values, $n_{L}$ and $n_{H}$, of the PSRF refractive index profile upon oxidation can be estimated using Eq. (4). We assume that oxidation proceeds uniformly through the thickness of the filter and employ Eq. (2) once again as well as the matrix method to calculate the reflectivity spectrum at different volumetric fractions, $x$, of the oxidized silicon. It is difficult to estimate how the thickness of each layer changes upon oxidation. The SEM data indicate that the oxide layer formed at the top of the filter is very thin $\sim 500 \mathrm{~nm}$ compared to the overall thickness of the filter and can be expected to impose no significant difference on the calculated results. Figure 9 shows the resonant wavelength change with the variation of the fraction of oxidized silicon. The resonant wavelength is taken as the center of the stop band obtained from the simulated spectra. Comparing these results with Fig. 6 allows an estimate of the volume fraction of silicon transformed to silicon dioxide of approximately $15 \%$ resulting from electrochemical oxidation. This volume density is in agreement with that roughly estimated from the AES measurements.

The change in $n_{L}$ and $n_{H}$ of the oxidized PSRF due to complete filling the pores with different liquids can be easily estimated from Eq. (4) by replacing the refractive index of air by that of the liquid concerned. The theoretical and experimental shifts of the resonant wavelength of the oxidized structure versus the refractive index of the liquid completely filling the pores are shown in Fig. 10. Surprisingly, theoretical results are less redshifted compared to the experimental

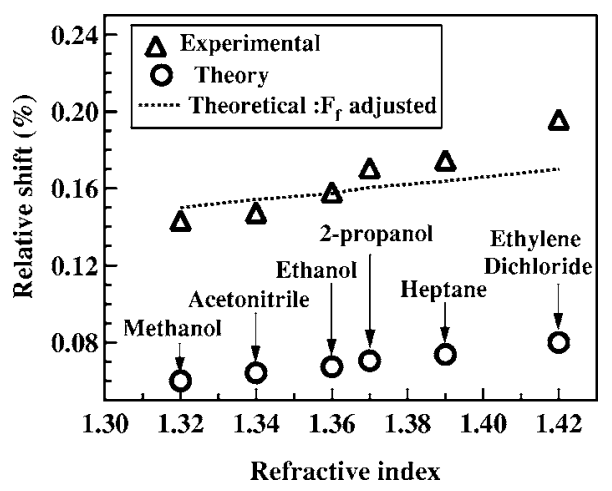

FIG. 10. Plot of the theoretical and observed relative shifts in resonant wavelength of anodically oxidized PSRF vs refractive index of the liquid immersed into the pores. 
case. This discrepancy may be attributed to our simplified assumptions of the layer-by-layer uniform pore filling with liquids as well as a homogeneous oxide formation through the thickness of the filter. In fact, there is no guarantee for a homogenous oxide formation through the whole thickness of the structure. The fraction of air remaining after oxidation, $F_{f}=P_{\text {in }}-1.27 x$, is supposed to suffer additional decrease if we consider the formation of inhomogeneous oxide. Taking this fraction as the fitting parameter, a decrease in the final porosity by $10 \%$ is needed to obtain a good fit between theory and experiment as indicated by the dotted line in Fig. 10. In all of the above calculations, we assumed that the oxidation of PSRF gives the form of silicon dioxide and neglected the possibility of the presence of silicon suboxides which may have a significant effect on the line shape of the oxidized structure. Stalmans et al., ${ }^{46}$ believed that the stoichiometric ratio between silicon and oxygen varies with the layer depth.

\section{CONCLUSIONS}

In this paper, we have used electrochemical oxidation as a convenient method to stabilize PSRFs employed for sensing gas-phase chemical species in air. This stabilization method improves the durability of the filter and prevents uncontrolled oxidation produced by ambient conditions. The oxidation behavior of porous silicon multilayers is similar to that of single layers. Electrochemical oxidation is selflimiting, and oxidizes the multilayer to an extent of about $15 \%$ confirmed by optical and AES measurements. The redshift due to liquid infiltration into the oxidized PSRF shows an approximately linear dependence on refractive index, as expected from the Bruggeman effective medium approximation.

\section{ACKNOWLEDGMENTS}

The authors would like to thank Dr. Y.-I. Suzuki, Uyemura Co. for his help with the AES measurements. One of the authors (M.J.S.) acknowledges funding support from the U.S. Air Force Office of Scientific Research (Grant No. F49620-02-1-0288) and the U.S. National Science Foundation (Grant No. DMR-0503006). This work was partially supported by a Grant-in-Aid from the Japan Society for the Promotion of Science under Grant No. 18350107.

${ }^{1}$ A. Uhlir, Bell Syst. Tech. J. 35, 333 (1956).

${ }^{2}$ L. T. Canham, Appl. Phys. Lett. 57, 1046 (1990).

${ }^{3}$ R. C. Anderson, R. S. Muller, and C. W. Tobias, Sens. Actuators, A 21, 835 (1990).

${ }^{4}$ S. Green and P. Kathirgsmsnsthsn, Mater. Lett. 52, 106 (2002).

${ }^{5}$ S. Zangooie, R. Jansson, and H. Arwin, J. Appl. Phys. 86, 850 (1999).

${ }^{6}$ V. S.-Y. Lin, K. Motesharei, K.-P. S. Dancil, M. J. Sailor, and M. R. Ghadiri, Science 278, 840 (1997).
${ }^{7}$ J. Drott, K. Lindström, L. Rosengren, and T. Laurell, J. Micromech. Microeng. 7, 14 (1997).

${ }^{8}$ K. L. Beattie, W. G. Beattie, L. Meng, S. L. Turner, R. Coral-Vazquez, D. D. Smith, P. M. McIntyre, and D. D. Dao, Clin. Chem. 41, 700 (1995).

${ }^{9}$ T. Laurell, J. Drott, L. Rosengren, and K. Lindström, Sens. Actuators B 31, 161 (1996).

${ }^{10}$ I. Ronga, A. Bsiesy, F. Gaspard, R. Hérino, M. Ligeon, F. Muller, and A. Halimaoui, J. Electrochem. Soc. 138, 1403 (1991).

${ }^{11}$ L. Pavesi and P. Mulloni, J. Lumin. 80, 43 (1999).

${ }^{12}$ G. Vincent, Appl. Phys. Lett. 64, 2367 (1994).

${ }^{13}$ M. Thönissen, M. G. Berger, W. Theiss, S. Hilbrich, M. Krüger, and H. Lüth, Solid State Phenom. 54, 65 (1997).

${ }^{14}$ P. A. Snow, E. K. Squire, P. St. J. Russell, and L. T. Canham, J. Appl. Phys. 86, 1781 (1999)

${ }^{15}$ T. Gao, J. Gao, and M. J. Sailor, Langmuir 18, 9953 (2002).

${ }^{16}$ S. Content, W. C. Trogler, and M. J. Sailor, Chem.-Eur. J. 6, 2205 (2000).

${ }^{17}$ H. Sohn, S. Letant, M. J. Sailor, and W. C. Trogler, J. Am. Chem. Soc. 122, 5399 (2000).

${ }^{18}$ B. G. Bovard, Appl. Opt. 32, 5427 (1993).

${ }^{19}$ M. Krüger et al., Thin Solid Films 297, 241 (1997).

${ }^{20}$ M. G. Berger et al., Thin Solid Films 297, 237 (1997).

${ }^{21}$ G. E. Jellison and H. H. Burke, J. Appl. Phys. 60, 841 (1986).

${ }^{22}$ S. M. Weiss, M. Molinari, and P. M. Fauchet, Appl. Phys. Lett. 83, 1980 (2003).

${ }^{23}$ M. Krüger, S. Hilbrich, M. Thönissen, D. Scheyen, W. Theiß, and H. Lüth, Opt. Commun. 146, 309 (1998).

${ }^{24}$ J.-N. Chazalviel and F. Ozanam, Mater. Res. Soc. Symp. Proc. 536, 155 (1999).

${ }^{25}$ V. Morazzani, M. Chamarro, A. Grosman, C. Ortega, S. Rigo, J. Siejka, and H. J. von Bardeleben, J. Lumin. 57, 45 (1993).

${ }^{26}$ N. Taghavinia, G. Lerondel, H. Makino, A. Parisini, A. Yamamoto, T. Yao, Y. Kawazoe, and T. Goto, J. Electrochem. Soc. 149, G251 (2002).

${ }^{27}$ D. Hamm, J. Sasano, T. Sakka, and Y. H. Ogata, J. Electrochem. Soc. 149, C331 (2002).

${ }^{28}$ W. Theiss, Adv. Solid State Phys. 33, 149 (1993).

${ }^{29}$ Y. Y. Li et al., Science 299, 2045 (2003).

${ }^{30}$ S. J. Gregg and K. S. W. Sing, Adsorption, Surface Area and Porosity, 2nd ed., (Academic, London, 1982), p. 112.

${ }^{31}$ K. H. Li, D. C. Diaz, J. C. Campbell, and C. Tsai, in Porous Silicon, edited by Z. C. Feng and R. Tsu (World Scientific Singapore 1994), pp- 261-274.

${ }^{32}$ J. E. Bateman, R. D. Eagling, B. R. Horrocks, A. Houlton, and D. R. Worrall, Chem. Commun. (Cambridge) 1997, 2275.

${ }^{33}$ M. G. Berger et al., Mater. Res. Soc. Symp. Proc. 358, 327 (1995).

${ }^{34}$ W. Theiß, M. Arntez, S. Hilbrich, M. Wernke, R. Arens-Fischer, and M. G. Berger, Phys. Status Solidi B 190, 15 (1995).

${ }^{35}$ H. Sugiyama and O. Nittono, J. Cryst. Growth 103, 156 (1990).

${ }^{36}$ G. Bomchil, A. Halimaoui, and R. Herino, Microelectron. Eng. 8, 293 (1988).

${ }^{37}$ A. Bsiesy, F. Gaspard, R. Herino, M. Leigeon, F. Muller, and J. C. Oberlin, J. Electrochem. Soc. 138, 3450 (1991).

${ }^{38}$ J. Y. Park and J. H. Lee, Res. Sci. Educ. 26, 315 (2004)

${ }^{39}$ A. Janshoff et al., J. Am. Chem. Soc. 120, 12108 (2002).

${ }^{40}$ R. Boukherroub, D. D. M. Wayner, and D. J. Lockwood, Appl. Phys. Lett. 81, 601 (1998).

${ }^{41}$ V. Mulloni and L. Pavesi, Appl. Phys. Lett. 76, 2523 (2000).

${ }^{42}$ K. Kaminska, T. Brown, G. Beydaghyan, and K. Robbie, Appl. Opt. 42, $4212(2003)$.

${ }^{43}$ M. Born and E. Wolf, Principle of Optics (Pergamon, Oxford, 1980).

${ }^{44}$ S. Billat, M. Thönissen, R. Arens-Fischer, M. G. Berger, M. Krüger, and H. Lüth, Thin Solid Films 297, 22 (1997).

${ }^{45}$ E. Lorenzo, C. J. Oton, N. E. Capuj, M. Ghulinyan, D. Navarro-Urrios, Z. Gaburro, and L. Pavesi, Phys. Status Solidi C 2, 3227 (2005).

${ }^{46}$ L. Stalmans, J. Poortmans, H. Bender, S. Jin, T. Conard, J. Nijs, L. Debarge, and A. Slaoui, J. Porous Mater. 7, 67 (2000). 